\title{
BEAM-PLASMA INTERACTION EXPERIMENTS AT THE UCLA NEPTUNE LABORATORY*
}

\author{
M. C. Thompson ${ }^{\dagger}$ C.E. Clayton, J. England, J. B. Rosenzweig, H. Suk, \\ UCLA, Los Angeles, CA 90024, USA
}

\begin{abstract}
We are currently planning several beam-plasma interaction experiments using the high quality $16 \mathrm{MeV}$ electron beam produced by the Neptune 1.625-cell photoinjector and PWT linac. The underdense $\left(n_{b}>n_{o}\right)$ plasma lens experiment will examine the focusing of a 4 $\mathrm{nC}$ beam of $30 \mathrm{psec}$ pulse duration as it passes through a thin $2 \mathrm{~cm}$ argon plasma lens [1]. We are also developing a negative $\mathrm{R}_{56}$ compressor beamline that will allow us to create ramped sub-picosecond beams of a shape well suited for driving large amplitude plasma wake fields and producing high transformer ratios. Simulations made using the 2-1/2 dimensional particle-in-cell code MAGIC indicate that we could use these ramped beams to produce fields of up to $10 \mathrm{GeV} / \mathrm{m}$ in a $10^{16} \mathrm{~cm}^{-3}$ plasma. Ramped beams are also suitable for driving plasma density gradient trapping experiments. When such a beam passes through a region where the plasma density drops suddenly the fast variation of the wake fields traps several $\mathrm{pC}$ of plasma electrons in the accelerating portion of the wake field.
\end{abstract}

\section{INTRODUCTION}

The UCLA Neptune beamline is operational and currently being used to study the physics of beam compression [2] and prepare for the plasma beat wave accelerator (PBWA) experiment [3]. Since the PBWA experiment is expected to occupy the present beamline for a considerable time, a second beamline will be built to accommodate our beam-plasma experiments. This new beamline will also serve as a negative $\mathrm{R}_{56}$ compressor.

One of the first beam-plasma experiments we plan to conduct on the second beamline will be a plasma density transition trapping experiment. In a plasma wake field accelerator (PWFA) a short, high density electron beam is used to drive large amplitude plasma waves. Accelerating gradients in these systems scale with the non-relativistic plasma frequency $\omega_{p}=\left(4 \pi n_{0} e^{2} / m_{e}\right)^{1 / 2}$, where $n_{\mathrm{o}}$ is the plasma density, $e$ is the electron charge, and $m_{e}$ is the electron mass. It follows that high gradient PWFA's have very short period waves. Accelerating a second beam in such a system typically requires injecting a subpicosecond beam into the drive beam's wake with subpicosecond timing accuracy. The difficultly of accomplishing this type of witness beam injection makes it desirable to develop a system in which charge is automatically load into the accelerating portion of the wake by the drive beam's interaction with its environment. Suk et al. [4] recently purposed a new selftrapping system for the use in the blow out regime of PWFA where $n_{b}>n_{\mathrm{o}}$ (underdense condition). In this scheme the beam passes though a sharp drop in plasma density where the length of the transition between the densities in region one (1) and region two (2) is smaller than the plasma skin depth $k_{p}^{-1}=v_{b} / \omega_{p}$, where $v_{b} \cong c$ the driving pulse's group velocity. As the drive beam's wake passes the sudden transition there is a period of time in which it spans both regions. The portion of the wake in region 2 has lower fields and a longer wavelength than the portion in region 1 . This means that the trajectory of region 2 plasma electrons near the transition is altered. As these electrons follow their normal trajectory away from the drive beam and backward toward the end of the wake they enter region 1 where the higher fields accelerate their oscillation and bring these electrons back to the axis sooner than the normal trajectory would have. When the parameters are correctly set, these rephrased electrons are inserted far enough into the accelerating region of the region 2 wake to be trapped and subsequently accelerated to high energy.

We plan to experimentally demonstrate plasma electron trapping at Neptune using a density transition in a relatively low density regime with $n_{\text {o }}$ on the order of $10^{13}$ $\mathrm{cm}^{-3}$. Conducting the first experiment using this selfinjection technique at low density greatly reduces the technical challenges associated with tailoring the plasma density at the cost of an acceptable reduction in acceleration gradient.

The optimum drive beam for plasma transition trapping is denser than the plasma in both regions and has a radius and length comparable to the plasma skin depth $k_{p}^{-1}$. This situation leads to unique challenges in different regions of the parameter space. In a dense plasma (e.g. $n_{\mathrm{o}}=10^{16}$ $\left.\mathrm{cm}^{-3}, k_{p}^{-1}=53 \mu \mathrm{m}\right)$ only a few $\mathrm{nC}$ are need, but this charge must be severely compressed in the radial and longitudinal directions. In a plasma of lower density (e.g. $n_{\mathrm{o}}=10^{13} \mathrm{~cm}^{-3}, k_{p}^{-1}=1.6 \mathrm{~mm}$ ), focusing and compression requirements are relaxed at the expense of the need for prodigious amounts of charge to maintain the drive beam at densities higher than the plasma. Our challenge at the Neptune Laboratory is to drive a $10^{13} \mathrm{~cm}^{-3}$ plasma with 5-6 $\mathrm{nC}$ and capture enough plasma electron charge to make a conclusive experimental observation.

* Work supported by U.S. Dept. of Energy grant DE-FG03-92ER40693.

† Email: matt@stout.physics.ucla.edu

Now working at Korea Electrotechnology Research Institute 


\section{PARTICLE-IN-CELL CODE SIMULATIONS}

In order to develop an experimental plan suitable to the capabilities of the Neptune Laboratory, extensive computer simulations are being performed with the particle-in-cell code MAGIC. Here we present simulation results for one well optimized case with parameters that are achievable at the Neptune Laboratory. The driving beam parameters of the simulation are shown in Table 1 and the plasma density profile is illustrated in Figure 1. The driving beam has a ramped longitudinal profile as shown in Figure 2. Ramped profiles of this type maximize the transformer ratio of the wake field [5] and can be produced using a negative $\mathrm{R}_{56}$ system as discussed in Sec. 3.2.

Table 1: Driving beam parameters.

\begin{tabular}{|l|c|}
\hline Beam Energy & $14 \mathrm{MeV}$ \\
\hline Beam Charge & $5.9 \mathrm{nC}$ \\
\hline Beam Duration (Ramped Profile) & $6 \mathrm{psec}$ \\
\hline Beam Density & $4 \times 10^{13} \mathrm{~cm}^{-3}$ \\
\hline Normalized Emittance & $30 \mathrm{~mm}-\mathrm{mrad}$ \\
\hline Beam Radius at Plasma Entrance & $540 \mu \mathrm{m}(\mathrm{rms})$ \\
\hline
\end{tabular}

As can been seen from Table 1 and Figure 1 the electron beam density is 2 times larger than the peak plasma density of $2 \times 10^{13} \mathrm{~cm}^{-3}$. The plasma density profile is tailored to maximize the amount of charge captured while maintaining an acceptable amount of acceleration. The first $\mathrm{cm}$ of the profile reflects a realistic finite rise time from zero to the maximum plasma density. After $5 \mathrm{~mm}$ of maximum density the transition takes place and the density is reduced to $18 \%$ of the maximum. This large drop is necessary to ensure adequate charge capture, which is achieved at the expense of reducing density and accelerating gradient in region 2.

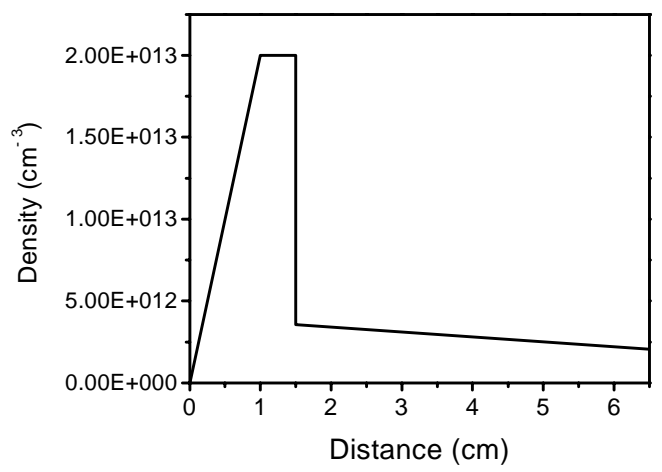

Figure 1: Plasma Density Profile.

In the simulation the transition is approximated as a perfect step function, the validity of this assumption will be elaborated on in Sec. 3.3. Finally, the gradual decline in plasma density after the transition slowly increases the size of the accelerating wake field region, which reduces the peak gradient but rephases the captured charge toward the peak field of the wake. This rephrasing both increases the amount of charge trapped and reduces energy spread.

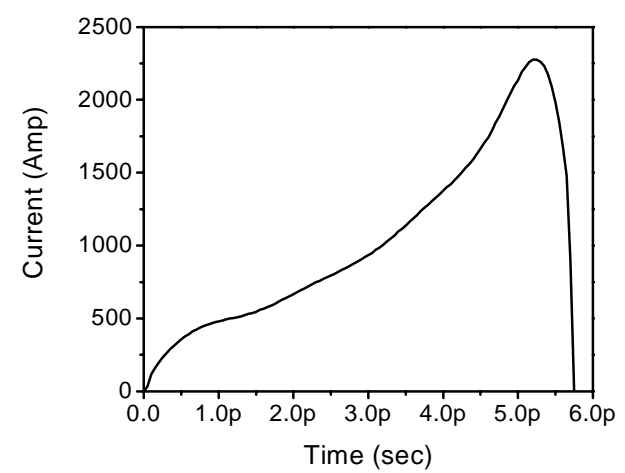

Figure 2: Drive beam longitudinal current profile.

Table 2: Captured plasma electron beam parameters.

\begin{tabular}{|l|c|}
\hline Beam Energy & $1.2 \mathrm{MeV}$ \\
\hline Energy Spread & $11 \%$ \\
\hline Beam Charge & $120 \mathrm{pC}$ \\
\hline Beam Duration & $4 \mathrm{psec}$ \\
\hline Beam Radius & $760 \mu \mathrm{m}$ \\
\hline Normalized Emittance & $40 \mathrm{~mm}-\mathrm{mrad}$ \\
\hline
\end{tabular}

The parameters of the bunch of captured plasma electrons are given in Table 2 and illustrated in Figure 3. The captured plasma electrons form a well defined beam of substantial charge that can be propagated and detected without major difficulty. The captured beam is also well separated from the drive beam in energy and should be easy to isolate.

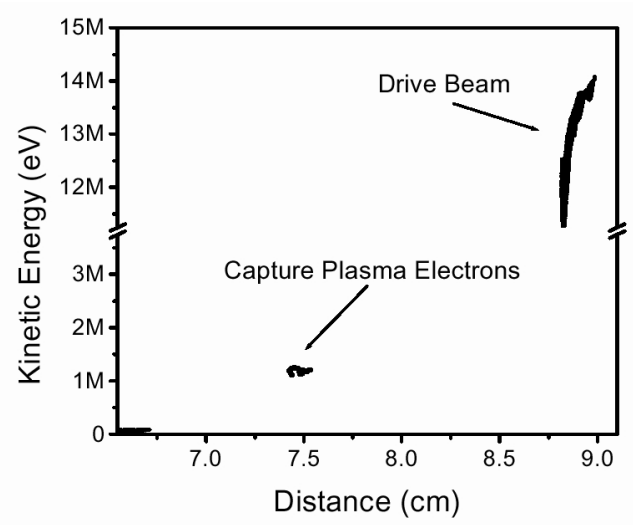

Figure 3: Kinetic energy versus longitudinal position for both plasma and drive beam electrons after they have emerged from the plasma.

\section{PLANNED EXPERIMENT}

The plasma trapping experiment will be done in the UCLA Neptune Laboratory. This beamline, which is based on a photocathode RF gun and PWT linac, routinely produces high quality beams for a variety of experiments. This section discusses the elements of this system as they relate to the purposed plasma trapping experiment. 


\subsection{Photoinjector and Linac}

In its current operating configuration the Neptune beamline produces approximately $500 \mathrm{pC}$ with $90 \mu \mathrm{J}$ of 266-nm UV in a 3 psec pulse using a single crystal copper cathode in a 1.625-cell photocathode RF gun. Although the charge can be increase somewhat by raising the accelerating gradient in the RF gun, and thereby enhancing the cathode quantum efficiency, this is insufficient to produce several $\mathrm{nC}$. Since the available UV laser energy is essentially fixed, we plan to the replace the current copper cathode with one made of magnesium in order to increase quantum efficiency.

\subsection{Negative $R_{56}$ Beamline}

As illustrated in Figure 4 we intend to build a second beamline to accommodate the plasma density transition trapping experiment. The new beamline will branch off of the existing beamline using the last two dipole magnets of the positive $\mathrm{R}_{56}$ compressor and bend back parallel using a set of identical dipole magnets. This beamline can be used a transport line or negative $R_{56}$ compressor depending on the momentum distribution of the beam. When operating in compressor mode the line can produce ramp distributions as shown in Figure 2.

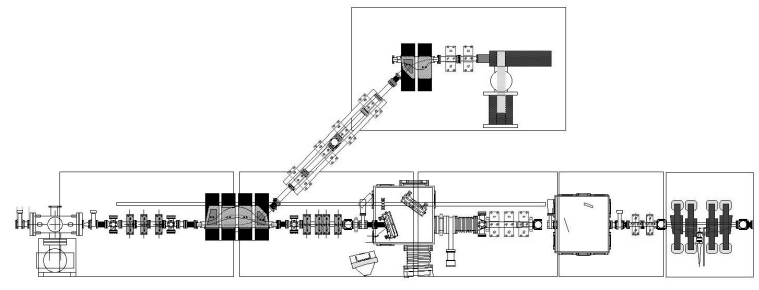

Figure 4: Illustration of the negative $\mathrm{R}_{56}$ beamline under construction at the Neptune Laboratory.

\subsection{Plasma Source}

The Neptune Laboratory has an argon cathode-anode pulse discharge plasma source that was originally design for the underdense plasma lens experiment. We intend to upgrade this source to make it suitable for a density transition trapping experiment while retaining the capability to perform the underdense plasma lens experiment [6] once the second beamline is complete.

The source has demonstrated operation in the mid $10^{12}$ $\mathrm{cm}^{-3}$ density range. We seek to increase the operating density of the device above $10^{13} \mathrm{~cm}^{-3}$ for the transition trapping experiment by increasing the operating temperature of its $\mathrm{LaB}_{6}$ cathode. Once the appropriate maximum density is achieved a density profile similar to that depicted in Figure 1 must be produced. In the discharge source plasma flows from the creation region near the cathode, through the hollow anode, and into the interaction region. The directionality of the flow allows the density to be manipulated by placing a metal mesh or line of vertical metal wires between the source region and the interaction point. If the density modifying obstruction is placed very close the drive beam path the transition in density should be sharp enough to show trapping. As illustrated in Figure 5, the transition only needs to be shorter than about $40 \%$ of the skin depth of region 2 for trapping efficiency to be comparable to that of a perfect step function. For the case shown in Section 2 any transition length under $1 \mathrm{~mm}$ is sufficient for effective trapping. We anticipate that sub-millimeter transition lengths will be achievable in a $10^{13} \mathrm{~cm}^{-3}$ density plasma with the masking techniques describe above.

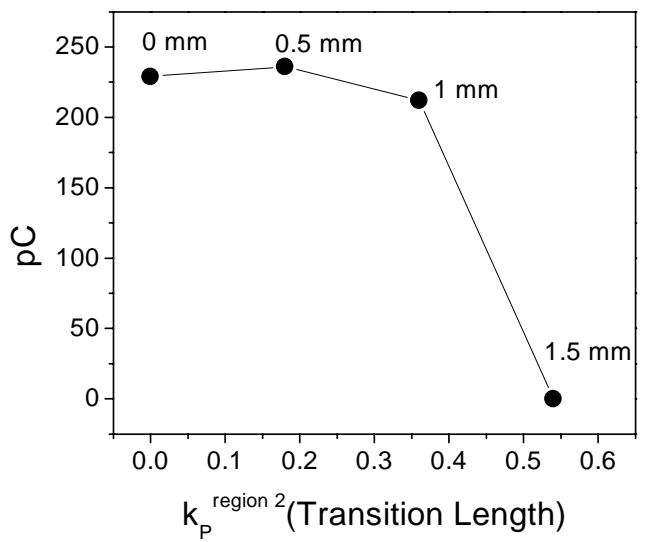

Figure 5: Dependence of the amount of charge captured on finite length of the plasma transition for the scenario describe in Section 2. Points are marked with the transition length.

\section{PRESENT STATUS AND CONCLUSIONS}

An experimental demonstration of plasma density transition trapping is achievable at the UCLA Neptune Laboratory and is being planned. The refinement of experimental parameters through simulation is ongoing. The major components of the negative $\mathrm{R}_{56}$ beamline are in hand awaiting a major lab shutdown for assembly. Work on upgrading the pulse discharge plasma source is ongoing and studies of density profile tailoring using plasma flow obstruction have recently begun. Work on improving the quantum efficiency of the Neptune 1.625cell RF gun continues as part of a larger UCLA program of advanced photocathode development.

\section{ACKNOWLEDGEMENTS}

The authors would like to thank T.C. Katsouleas for his assistance with the MAGIC PIC simulations.

\section{REFERENCES}

[1] H. Suk, et al., PAC Proceeding, 3708 (1999)

[2] S. Anderson, et al., "Commissioning of the Neptune Photoinjector," these proceedings.

[3] C. Clayton, et al., Nucl. Instr. Methods A 410, 378 (1998).

[4] H. Suk, et al., Phys. Rev. Lett. 86, 1011 (2001).

[5] P. Chen, et al., Stanford Linear Accelerator Center Report No. SLAC-PUB-3731 (1985).

[6] H Suk, et al., IEEE Trans. Plasma Sci. 28, 271 (2000). 